\title{
Low Back Ache Treatment with Botulinum Neurotoxin Type A
}

\author{
Local Experience in Kuwait
}

\author{
Venkatesan Nagarajan ${ }^{a} \quad$ Asmahan Al-Shubailia ${ }^{a}$ Yasser M. Ayad ${ }^{a}$ \\ John Alexander ${ }^{a} \quad$ Khadijah Al-Ramezi $^{b}$ \\ Departments of a Neurology and ${ }^{b}$ Physiotherapy, Ibn Sina Hospital, Kuwait, Kuwait
}

\author{
Key Words \\ Botox - Botulinum toxin - Chronic back pain - Kuwait • \\ Muscle spasm $\cdot$ Nociceptive pain
}

\begin{abstract}
Objective: To investigate the efficacy, safety and tolerability of paraspinal administration of botulinum neurotoxin type A (BoNT-A) in patients with chronic low back ache (LBA). Subjects and Methods: Eight patients with chronic LBA were injected with BoNT-A at three sites on either side of lumbar paraspinal muscles. The patients rated their pain intensity using a visual analogue scale (VAS) from 0 to 10, and our physiotherapist assessed the paraspinal muscle spasm using a functional scale (FS) from 0 to 5 . The improvement in both VAS and FS 30, 60 and 90 days after BoNT-A from baseline was analyzed separately using paired $t$ test. The correlation between the muscle spasm (FS) and pain relief (VAS) was analyzed using the Spearman's rank correlation coefficient test. The level of statistical significance was $p<0.05$. Results: Five (63\%) patients showed a remarkable recovery in VAS and FS, whereas 2 (25\%) patients showed improvement only in FS. Statistically significant improvement was achieved in VAS and FS independently 30 days $(p<0.02$ and $p<0.02$, respectively), 60 days ( $p<0.01$ and $p<0.001$, respectively) and 90 days ( $p<0.02$ and $p<0.001$, respectively) after treatment. Pain relief started early and it was independent of relief of muscle spasm 30 days after treatment $(r=0.685 ; p>$
\end{abstract}

0.05). Conclusion: With this limited study, we have demonstrated that the paraspinal injection of BoNT-A is effective in relieving chronic LBA without producing side effects. The injection is an easy procedure, well tolerated and did not require anesthesia or imaging technique.

Copyright @ 2007 S. Karger AG, Basel

\section{Introduction}

Low back ache (LBA) is the price man has to pay for assuming erect posture [1]. Other than common cold, LBA is the most common disease affecting manhood [2]. Almost $80 \%$ of the population will experience LBA at least once in their lifetime, and the incidence is increasing steadily due to a sedentary lifestyle and lack of exercise [3]. The majority (90\%) of people suffer from mechanical LBA which arises out of sprain or strain of muscles, tendons, ligaments or involvement of bony attachments [3]. Though mechanical LBA is usually a self-limiting condition, sometimes the initial injury which initiated this nociceptive response would heal, but the reflex muscle spasm persists leading to chronic LBA as sketched in figure 1 . The paraspinal muscle spasm is primarily implicated in chronic LBA [4]. There are several therapeutic modalities for reducing reflex muscle spasm such as slow stretch exercise, massage, muscle-relaxant medication and biofeedback [5]. These therapeutic modalities have 


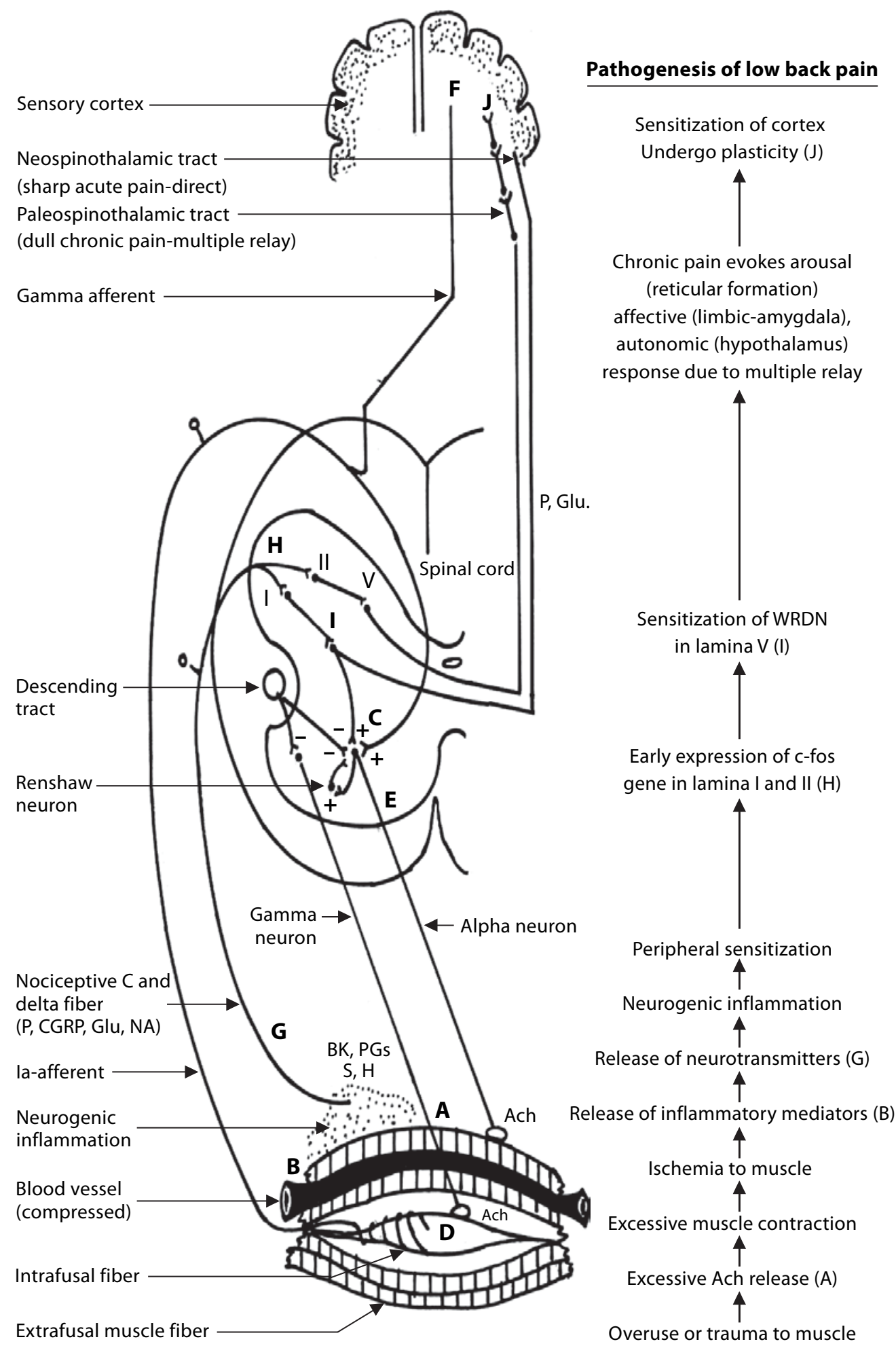

Fig. 1. Pathogenesis of LBA and possible sites of action of BoNT A-J (description given in a tabular form in table 1). 
Table 1. Site of action of BoNT (A-J as marked in fig. 1)

\begin{tabular}{llll}
\hline Neurotransmitter blocked & Site & Effect & Consequences \\
\hline Ach & alpha neuron & $\begin{array}{l}\text { paralyzes injected } \\
\text { muscles (A) }\end{array}$ & $\begin{array}{l}\text { No muscle ischemia or release of inflammatory media- } \\
\text { tors (B); no reflex stimulation of alpha neuron by acute } \\
\text { pain circuit (C). } \\
\text { By negative influence over alpha neuron through gam- } \\
\text { ma loop (E), it paralyzes non-injected muscles; alters } \\
\text { sensory processing through supraspinal projection (F). }\end{array}$ \\
$\begin{array}{l}\text { Pain-modulating neuropep- } \\
\text { tides in C and delta fibers }\end{array}$ & $\begin{array}{l}\text { nociceptor and } \\
\text { spinal ganglion } \\
\text { spindle (D) }\end{array}$ & $\begin{array}{l}\text { no firing of } \\
\text { nociceptors }(\mathrm{G})\end{array}$ & $\begin{array}{l}\text { No early expression c-fos gene (H); desensitize WRDN } \\
\text { in lamina } \mathrm{V}(\mathrm{I}) \text { and reduce the expanded map of sensi- } \\
\text { tized cortex (J). }\end{array}$ \\
\hline
\end{tabular}

Pain-modulating neuropeptides are substance $\mathrm{P}(\mathrm{P})$, glutamate $(\mathrm{Glu})$, calcitonin gene-related peptide $(\mathrm{CGRP})$ and nor-adrenaline (NA). Inflammatory mediators are bradykinin (BK), prostaglandins (PGs), serotonin $(\mathrm{S})$ and acidic $\mathrm{pH}\left(\mathrm{H}^{+}\right)$. Ach $=\mathrm{Acetylcholine}$; WRDN = wide-range dynamic neuron.

several disadvantages that include cost, side effects and ineffectiveness to relieve the symptoms for a longer period of time. Local injection of botulinum neurotoxin type A (BoNT-A) has been shown to selectively weaken the painful muscles for a lasting response, thereby breaking the vicious cycle of muscle spasm-pain-spasm and hence allows the subject to participate in a physical exercise program for a long-term recovery [5].

BoNT is an exotoxin produced by the Gram-positive anaerobic bacterium, Clostridium botulinum, which inhibits the release of acetylcholine at the neuromuscular junction, leading to a life-threatening paralytic illness. Among seven serotypes A, B and E are potent human neurotoxins. This most powerful biological toxin known to mankind is now used as a weapon against hyperactive muscles in spasticity, dystonia and different forms of muscle spasm including blepharospasm [6]. BoNT-A not only controls the muscle spasm but is also effective in relieving the pain by different mechanisms of action. The neuromuscular effect of BoNT is due to blockade of acetylcholine from $\alpha$ and $\gamma$ motor fibers, whereas the antinociceptive effect is mainly due to blockade of pain-modulating neurotransmitters (glutamate, substance P, calcitonin gene-related peptides and noradrenaline) from sensory nerve endings and ascending tracts [7-10]. The possible sites of action of BoNT-A are drafted in figure 1 and table 1.

Since US Federal Drug Administration's approval in 1981 for strabismus, the spectrum of therapeutic application of BoNT is ever expanding [6]. Relief of pain and spasm was observed in the myofascial pain syndrome, which resembles LBA, by injecting BoNT-A in trigger points $[5,11]$. Successes in these conditions have led to the use of BoNT in chronic LBA. But there are only few studies on the paraspinal administration of BoNT-A in chronic LBA [12-14], hence we investigated the efficacy, safety and tolerability of paraspinal administration of BoNT-A in selected chronic LBA patients.

\section{Patients and Methods}

Eight patients, 5 males and 3 females, aged 28-70 years, from the Neurology and Neurosurgical Outpatient Service were recruited for the study. Their characteristics at the beginning of the study are given in table 2 . Inclusion criteria were existence of chronic LBA lasting $>6$ months associated with paraspinal muscle spasm and failure to respond to conservative management of administration of analgesics, antidepressant, muscle relaxants and physical therapy. Exclusion criteria were acute LBA, systemic diseases such as cancer, spinal infection and inflammatory arthritis, progressive or persistent neurological signs due to herniated disk or spinal stenosis, previous spinal surgery and administration of local anesthetics or corticosteroids or BoNT-A in the lumbar paraspinal muscles.

To evaluate the isolated effect of BoNT-A on LBA, no physical therapy was performed on the patients during the study period. If the patients were on physiotherapy it was phased out and all the muscle-relaxant medications including Lioresal were slowly tapered and stopped 6 weeks prior to injection. The pain killers and/or antidepressants were continued as such without changing the dosage. No new drugs were allowed till the final assessment.

The paraspinal muscle injection was done in the Outpatient Department after carefully explaining the procedure to the patient. The freeze-dried powder of BoNT-A (Botox, Allergan, 100 
Table 2. The patients' characteristics at the beginning of the study

\begin{tabular}{lllllllll}
\hline No. & $\begin{array}{l}\text { Age } \\
\text { years }\end{array}$ & Sex & $\begin{array}{l}\text { LBA } \\
\text { duration } \\
\text { years }\end{array}$ & $\begin{array}{l}\text { X-ray } \\
\text { LSS }\end{array}$ & $\begin{array}{l}\text { MRI } \\
\text { LSS }\end{array}$ & NCS & VAS & FS \\
\hline 1 & 28 & M & 1 & N & N & N & 8 & 3 \\
2 & 35 & F & 1.6 & N & CDB & N & 6 & 3 \\
3 & 70 & F & 10 & LS & N & N & 7 & 3 \\
4 & 45 & M & 2.3 & N & CDB & N & 8 & 3 \\
5 & 29 & M & 0.6 & N & N & N & 7 & 3 \\
6 & 39 & F & 1.8 & N & N & N & 10 & 5 \\
7 & 32 & M & 3.2 & N & N & N & 10 & 4 \\
8 & 42 & M & 4.3 & N & N & N & 9 & 3 \\
\hline
\end{tabular}

MRI = Magnetic resonance imaging; NCS = nerve conduction studies; LSS = lumbosacral spine; LS = lumbar spondylosis; $\mathrm{CDB}=$ central disk bulge (in both cases the level was L4-L5); $\mathrm{N}=$ normal.

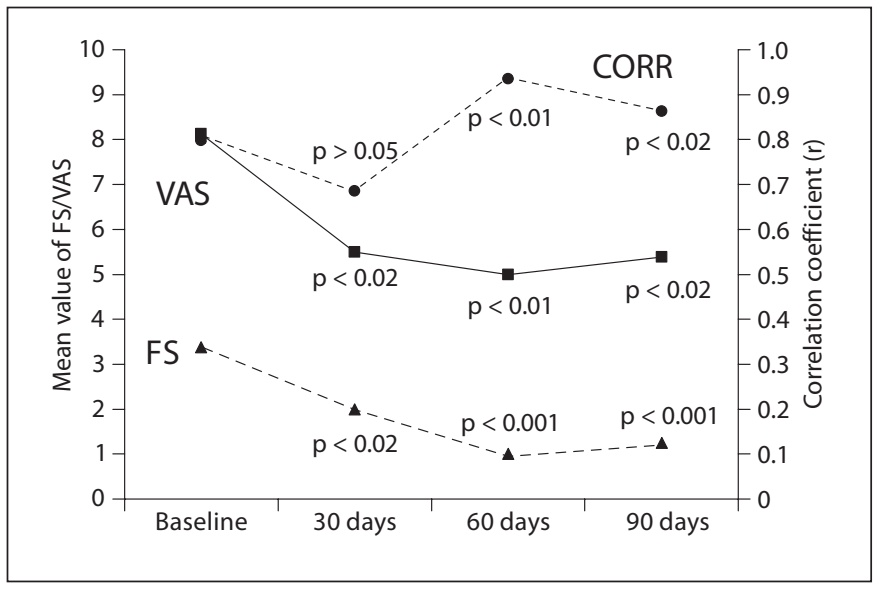

Fig. 2. Mean improvement in VAS and FS and $r$ value of the correlation (CORR) between the muscle spasm (FS) and the pain relief (VAS). The mean values and their respective $\mathrm{p}$ value are given for baseline, and 30, 60 and 90 days after treatment. units per vial) was dissolved in $4 \mathrm{ml}$ of preservative-free $0.9 \%$ saline, so that each milliliter contained 25 units. Shaking the vial was avoided while reconstituting and the solution was used immediately. For each patient, a standard dose of 300 units was prepared and loaded in six 2-ml syringes fitted with a 22-gauge needle. While the patient was in prone position, 50 units of BoNT were injected into the paraspinal muscle two inches apart in three sites on either side of lumbar vertebrae between L2 and L5. Care was taken to inject the toxin into the core of the muscles at the same time avoiding its spillover into the vascular compartment. The patient was encouraged to report any untoward side effect during the injection and subsequent follow-up.

The patients rated pain intensity using a visual analogue scale (VAS) which was equally graded from 0 to 10 on a straight line; 0 being no pain and 10 being worst pain ever experienced by the patient. Physiotherapists blinded to the study assessed palpable muscle spasm that is firmness and not tenderness and the range of movements (ROM) using a functional scale (FS) that was graded from 0 to 5 ; 0 : none, 1 : mild muscle spasm, 2 : moderate spasm with normal ROM, 3: severe muscle spasm with reduced ROM, 4: severe muscle spasm with marked limitation of ROM and 5: severe muscle spasm with postural deviation or fixed posture. Both assessments were done at baseline and 30, 60 and 90 days after the injection.

The primary outcome variables were determined by reduction of pain in VAS and muscle spasm in FS. Significant improvement was defined as a reduction of two grades in FS and 50\% in VAS from baseline. The improvement in both VAS and FS 30, 60 and 90 days after BoNT-A injection was analyzed separately using paired t test. The correlation between the muscle spasm (FS) and the pain relief(VAS) was analyzed using the Spearman's rank correlation coefficient test. The level of statistical significance was $\mathrm{p}<0.05$.

\section{Results}

The patients tolerated the procedure well. Anaphylactic reactions were not observed during the procedure. There was no local inflammatory reaction, pain or swelling other than the pain during injection. No flu-like symptom, excessive muscle weakness or fatigue was reported during follow-up.

Five $(63 \%)$ patients showed a remarkable improvement in VAS and FS. In these 5 patients, the improvement was observed from the first to the last visit, but the maximum response was seen in the second visit. They showed $50 \%$ reduction in the VAS score in the 1st month, which improved further in the 2nd month to reach the maximum response. Thereafter, the pain score slightly increased in the 3rd month but was still lower than at baseline and 1 month after treatment. In FS, a two-grade reduction was seen only in 2 patients in the 1st month but in all 5 patients by the 2 nd month. The same improvement was maintained in the $3 \mathrm{rd}$ month as well.

Among the 3 (37\%) non-responders, 2 patients reported no improvement in VAS but showed a reduction of two grades in FS, which was maintained throughout the follow-up. One patient reported worsening of LBA after BoNT-A injection but did not show any change in FS even though he had only a moderate degree of muscle spasm.

For all 8 patients, the improvement in VAS and FS compared to baseline is shown in figure 2. Overall there was a statistically significant improvement in VAS and FS 
independently 30 days $(p<0.02$ and $p<0.02$, respectively), 60 days ( $\mathrm{p}<0.01$ and $\mathrm{p}<0.001$, respectively) and 90 days ( $p<0.02$ and $p<0.001$, respectively) after treatment. There was a good correlation between the intensity of pain (VAS) and muscle spasm (FS) at baseline $(\mathrm{r}=0.798$ and $\mathrm{p}<0.05), 60$ days $(\mathrm{r}=0.935$ and $\mathrm{p}<0.01)$ and 90 days $(\mathrm{r}=0.863$ and $\mathrm{p}<0.02)$ but not 30 days $(\mathrm{r}=0.685$ and $\mathrm{p}>0.05$ ) after treatment (fig. 2).

\section{Discussion}

Though our study population was small, the BoNT injection into the paraspinal muscles in a single sitting produced a marked reduction in muscle spasm and pain intensity in chronic LBA patients. Pain relief occurred early before the reduction in muscle spasm reached its maximum. This is consistent with the view expressed by Aoki et al. [7].

Even though LBA is produced by the paravertebral muscle over activity, there is a complex interplay of social and psychological factors in the sustenance of the chronic pain status [15]. Improvement may not be achieved without addressing this concern. Possibly this may be the reason in 2 non-responders who had clear reduction in muscle spasm but not in pain relief. Though the antibody formation is implicated for ineffectiveness of BoNT after repeated injection, occasionally some may not respond to the first injection [16]. Probably our 3rd non-responder falls into that category.

In any chronic pain syndrome, complete relief of symptoms may not be achieved. So the therapeutic goals should focus on improving the quality of life and optimizing the day-to-day function. Most of our patients reported improvements in the activities of daily living after BoNT injection. As pain is complex subjective experience evoking an emotional response, objective quantification is very difficult. Subjective VAS, used in several studies including this one, is a more reliable measure of pain [17]. Stretching exercise after BoNT is known to improve the long-term outcome and reduce the need for reinjection [11]. In this study, we avoided physical therapy and the use of muscle relaxant in order to document the independent effect of BoNT in relieving the spasm.

Our findings are similar to previous reports [12-14]. In a retrospective study of 51 randomly selected patients, Carrasco et al. [12] found there was a greater reduction in pain and pain-free time with BoNT-A than with an anesthetic-steroid combination. Apart from the limitation of a retrospective study, they injected gluteus maxi- mus and quadratus lumboram other than paraspinal muscles and used electromyographic guidance to inject trigger points. We injected only paraspinal muscles and did not use electromyography, ultrasonography and CT for guidance, and did also not use local or general anesthesia for pain.

In a randomized, double-blind study of unilateral administration of BoNT-A or saline in the paraspinal muscles of 31 chronic LBA patients, Foster et al. [13] found a significant improvement (60 vs. $12.5 \%$ ) in the BoNTtreated group at 8 weeks. Unlike this study we did not look for any specific tender points or the side of maximum involvement, and our patients received BoNT-A on both sides. Based on the principle that BoNT can spread locally we have limited the needle prick to three sites on either side in contrast to five sites in their study.

Jabbari et al. [14] studied the beneficial effect of repeated paraspinal BoNT-A injection over 14 months on a heterogeneous group of 75 patients with chronic LBA. The study included 20 patients with root pain, 22 with focal sensory loss, 14 with failed back syndrome after surgery and 15 patients after epidural injections. Moreover, 95\% of their patients had lumbar MRI abnormalities which included chronic disk protrusion, canal narrowing and stenosis, degenerative changes or a combination of any of these structural changes. After the first injection, $52 \%$ of patients had significant pain relief at 2 months, and among these responders, $91 \%$ continued to respond till the end of the study period (14 months). Adverse side effects were reported in $4 \%$ of their patients during repeated injection and not after the first dose. This may be the reason for the lack of side effects observed in our patients. Apart from this study, Difazio and Jabbari [15], in a review of 7 patients who received repeated BoNT-A every 3-4 months for 1-3 years, showed a sustained improvement. Follow-up was not long enough to see the weaning effect of the toxin in our patients.

\section{Conclusion}

BoNT injection of paraspinal muscles is a safe and simple office procedure, which is well tolerated and does not require supportive staff, anesthesia or modern gadgets. Though this study was done with a small number of selected patients with chronic LBA, it showed statistically significant improvements in pain and muscle spasm. However, larger controlled blinded studies are required before integrating this concept of treatment into the multimodal therapeutic approach to chronic LBA. 


\section{References}

1 Giles LGF: Introduction; in Giles LGF, Singer KP (eds): Clinical Anatomy and Management of Low Back Pain. Oxford, Butterworth Heinemann, 1997, pp 3-17.

2 Jabbari B, Ney J: Treatment of low back pain with botulinum neurotoxins. Pain Pract 2004;4(suppl 1):S47-S53.

$>3$ Deyo RA, Weinstein JN: Low back pain. N Engl J Med 2001;344:363-370.

4 Sherman RA: Relationships between strength of low back muscle contraction and reported intensity of chronic low back pain. Am J Phys Med 1985;64:190-200.

$\checkmark 5$ Cheshire WP, Abashian SW, Mann JD: Botulinum toxin in the treatment of myofascial pain syndrome. Pain 1994;59:65-69.

$\checkmark 6$ Jankovic J: Botulinum toxin in clinical practice. J Neurol Neurosurg Psychiatry 2004;75: 951-957.
7 Aoki KR: Evidence for antinociceptive activity of botulinum toxin type A in pain management. Headache 2003;43(suppl 1):S9S15.

8 Mense S: Neurobiological basis for the use of botulinum toxin in pain therapy. J Neurol 2004;251(suppl 1):I1-I7.

$\checkmark 9$ Hallet M: How does botulinum toxin work? Ann Neurol 2000;48:7-8.

10 Silberstein SD: Neurotoxins in the neurobiology of pain. Headache 2003;43(suppl 1) S2-S8.

11 Porta M: A comparative trial of botulinum toxin type-A and methylprednisolone for the treatment of myofascial pain syndrome and pain from chronic muscle spasm. Pain 2000;85:101-105.

12 Carrasco AT, Wescoat L, Roman A: A retrospective review of botulinum toxin type $A$ compared with standard therapy in the treatment of lumbar myofascial back patients. Pain Clin 2003;15:205-211.
13 Foster L, Clapp L, Erickson M, Jabbari B Botulinum toxin $\mathrm{A}$ and chronic low back pain: a randomized, double-blind study. Neurology 2001;56:1290-1293.

14 Jabbari B, Ney J, Sichani A, Monacci W, Foster L, Difazio M: Treatment of chronic low back pain with botulinum neurotoxin A: an open label, pilot study. Pain Med 2006;7: 260-264.

15 Difazio M, Jabbari B: A focused review of the use of botulinum toxins for low back pain. Clin J Pain 2002;18:S155-S162.

16 Thant ZS, Tan EK: Emerging therapeutic ap plications of botulinum toxin. Med Sci Monit 2003;9:RA40-RA48.

17 Salo D, Eget D, Lavery RF, Garner L, Bernstein S, Tandon K: Can patients accurately read a visual analog pain scale. Am J Emerg Med 2003;21:515-519. 